\title{
The Neurasthenic Nipper-Juvenile Idiopathic Arthritis
}

\author{
Anubha Bajaj* \\ Consultant Histopathologist, AB Diagnostics, India.
}

Correspondence to: Anubha Bajaj, Consultant Histopathologist, AB Diagnostics, India; E-mail: anubha.bajaj@gmail.com Received date: January 15, 2021; Accepted date: January 27, 2021; Published date: February 04, 2021

Citation: Bajaj A (2021) The Neurasthenic Nipper-Juvenile Idiopathic Arthritis. J Clin Biomed Invest 1(1): pp. 1-6. doi: 10.52916/jcbi214003

Copyright: (C2021 Bajaj A. This is an open-access article distributed under the terms of the Creative Commons Attribution License, which permits unrestricted use, distribution and reproduction in any medium, provided the original author and source are credited.

\section{ABSTRACT}

Juvenile Idiopathic Arthritis (JIA) is a heterogeneous group of paediatric, idiopathic, inflammatory arthritis exceeding >six weeks duration and commonly arising in children beneath $<16$ years. International League of Associations for Rheumatology (ILAR) has categorized juvenile idiopathic arthritis into distinctive subclasses as pauciarticular variant or oligoarthritis, Rheumatoid Factor (RF) positive polyarthritis, Rheumatoid Factor (RF) negative polyarthritis, systemic arthritis, psoriatic arthritis, enthesitis-related arthritis and undifferentiated arthritis.

The condition is posited to arise from environmental factors, viral or bacterial infection or demonstrates a genetic predisposition. Juvenile idiopathic arthritis depicts decimated joint function with reduced Range of Motion (ROM), joint pain, morning stiffness, limping due to pain in the lower extremities, joint deformity and joint swelling commonly discerned within the knee, hand or foot, anomalous limb growth with leg length discrepancies, uveitis, reoccurring pyrexia, cutaneous rash, myalgia, weight loss and disorders of skeletal growth. An intense, synovial infiltration of T lymphocytes, B lymphocytes, plasma cells, macrophages and dendritic cells is observed along with villous hyperplasia and hypertrophy, endothelial activation and hyperplasia and hyperplasia of synoviocytes.

\section{Keywords}

Juvenile idiopathic arthritis, Lymphocytes, Juvenile rheumatoid arthritis, Leukocyte

\section{Introduction}

Juvenile Idiopathic Arthritis (JIA) is comprised of a heterogeneous group of paediatric, idiopathic, inflammatory arthritis in association with exclusion of various categories of chronic arthritis. Juvenile idiopathic arthritis denominates an arthritis commonly exceeding $>$ six weeks duration arising in children beneath $<16$ years. Juvenile idiopathic arthritis is devoid of specific investigations for cogent disease discernment and prediction of disease activity. A child with persistent limp following minor trauma requires evaluation for conditions such as juvenile idiopathic arthritis or leukaemia. Additionally, paediatric chronic arthritis or juvenile idiopathic arthritis demonstrates an evolutionary terminology of juvenile chronic arthritis (JCA) or juvenile rheumatoid arthritis (JRA). Distinctive categorization of juvenile idiopathic arthritis is associated with specific phenotypes, genetic predisposition, pathogenesis, biochemical parameters, biological course and prognostic outcomes.

\section{Disease Characteristics}

Juvenile idiopathic arthritis is a frequently discerned paediatric rheumatic disease of the Western world with a variable incidence of 1.6 to 23 per 100000 children. Possible occurrence of juvenile idiopathic arthritis in siblings is enhanced by 15 times to 30 times. Juvenile idiopathic arthritis usually implicates children below $<16$ years with a mean age of disease onset at 1 year to 3 years. A racial predilection is absent. The heterogeneous disorder with diverse subtypes demonstrate synovitis as a common feature $(1,2)$. Majority of juvenile idiopathic arthritis demonstrate a female predominance with a female to male proportion of 2:1. Enthesitis-related arthritis depicts a male preponderance and the systemic variant is accompanied by an equivalent gender distribution(1,2). Autoimmune juvenile idiopathic arthritis is classified contingent to number of implicated joints and pertinent clinical signs or symptoms. International League of Associations for Rheumatology (ILAR) has categorized juvenile idiopathic arthritis into distinctive subclasses as

a) Pauciarticular variant or oligoarthritis ( $50 \%$ to $60 \%$ )

b) Rheumatoid Factor (RF) positive polyarthritis ( $2 \%$ to $7 \%$ )

c) Rheumatoid Factor (RF) negative polyarthritis ( $11 \%$ to $28 \%$ )

d) Systemic arthritis ( $10 \%$ to $20 \%$ )

e) Psoriatic arthritis ( $2 \%$ to $15 \%$ )

f) Enthesitis-related arthritis ( $1 \%$ to $7 \%$ )

g) Undifferentiated arthritis $(1,2)$

Chronic arthritis is a consistent component of aforesaid subtypes wherein extra-articular and systemic manifestations are pertinent to diverse categories $(1,2)$.

\section{Disease Pathogenesis}

Of obscure aetiology, juvenile idiopathic arthritis is posited to arise from environmental factors, viral or bacterial infection or demonstrates a genetic predisposition. Essentially perceived as an autoimmune disease, immune cells and auto-antibodies invade the synovium with consequent inflammation, thickening and aberrant growth. Anomalous synovial growth damages adjacent bone, joint cartilage and circumscribing soft tissues $(1,2)$. Besides, the systemic variant is contemplated to be an auto-inflammatory disorder. Chronic arthritis accompanying juvenile idiopathic arthritis demonstrates aberrant immune 
responses in susceptible children concurrent to interaction between environmental and genetic factors $(3,4)$.

Environmental factors such as caesarean section babies or exposure to antibiotics may initiate the arthritic process whereas disease emergence in siblings or breastfeeding appears protective $(3,4)$.

Involvement of microorganisms such as parvovirus B19, Epstein Barr virus, salmonella spp, chlamydia pneumoniae and streptococcus remains debatable $(3,4)$. Genetic factors are significant in representation of juvenile idiopathic arthritis and around $25 \%$ to $40 \%$ instances may appear in monozygotic twins $(3,4)$.

Human Leukocyte Antigen (HLA) and non Human Leukocyte Antigen (non HLA) alleles appear concordant to diverse subtypes of juvenile idiopathic arthritis and uveitis. Especially, HLA-A2, HLA-DRB1:11 and HLA-DRB1:08 appear concordant to oligoarticular and Rheumatoid Factor (RF) negative polyarticular juvenile idiopathic arthritis.

HLADRB1:01 and HLADRB1:04 appear to be associated with Rheumatoid Factor (RF) positive polyarthritis $(3,4)$.

HLADRB1:04 and DRB1:11 accompany systemic juvenile idiopathic arthritis. HLA-B27:05 and HLA-B27:04 are frequently discerned HLA-B27 subtypes associated with Enthesitis-Related Arthritis (ERA).

HLADRB1:01 and DQA1:01 are concurrent with the psoriatic variant whereas HLADRB1:11 and HLADRB1:13 accompany uveitis. Extensive incrimination of HLA-DR alleles predispose to preliminary occurrence of juvenile idiopathic arthritis $(3,4)$. Imbalance between regulatory T cells interferon gamma (IFN-Ỹ) secreting Thelper cells (Th1) and interleukin 17 (IL-17) secreting Thelper cells (Th17) engendering adaptive immunity is manifest between majority of variants of juvenile idiopathic arthritis. Interleukin 17 (IL-17) generates pro-inflammatory cytokines and matrix metalloproteinases with consequent occurrence of joint injury in oligoarthritis, polyarthritis and psoriatic arthritis $(3,4)$.

Enthesitis Related Arthritis (ERA) secretes a crucial cytokine interleukin 23 (IL-23) which engenders inflammation through activation of interleukin 17 (IL-17) and Tumour Necrosis Factor (TNF) whereas configuration of new bone occurs through interleukin 22 (IL-22).

Systemic arthritis demonstrates a persistent activation of innate immunity with concurrence of monocytes, macrophages and neutrophils. Consequently, innate pro-inflammatory cytokines such as interleukin 1 beta (IL-1ß), interleukin 6 (IL-6) and interleukin 18 (IL-18) may induce clinical symptoms of systemic arthritis $(3,4)$.

\section{Clinical Elucidation}

Majority of subtypes of juvenile idiopathic arthritis depict periods of remission with exacerbation, decimated joint function with reduced range of motion (ROM), joint pain, morning stiffness, limping due to pain in the lower extremities, joint deformity and joint swelling commonly discerned within the knee, hand or foot, anomalous limb growth with leg length discrepancies, uveitis, reoccurring pyrexia, cutaneous rash, myalgia, weight loss and disorders of skeletal growth $(5,6)$. Juvenile idiopathic arthritis is associated with unpredictable biological behaviour (Figure 1-7).
Self-limiting disease may ensue apart from an unremitting disease process with enhanced possibility of joint destruction. Juvenile idiopathic arthritis usually depicts symptoms of inflammatory joint disease such as synovitis, joint effusion, soft tissue swelling, osteopenia, bone oedema and bone erosions. Additional clinical representation is contingent to developmental age, imbalance of epiphyseal growth plate, premature epiphyseal closure and limb length discrepancies $(5,6)$. Specific clinical manifestations, values of Rheumatoid Factor (RF) and HLA-B27 are adopted to categorize subtypes of juvenile idiopathic arthritis as

- Oligoarthritis which is commonly discerned and denominated as chronic arthritis implicating $\sim 4$ or $<4$ joints for a duration of six months. Female subjects below $<8$ years are usually implicated and demonstrate swollen joints, limb or gait anomalies with leg length discrepancy. Generally, asymmetrical involvement of large joints such as knee, elbow, wrist, ankle, shoulder or hip is observed. Around $\sim 20 \%$ to $30 \%$ children develop uveitis. Persistent oligoarthritis is associated with implication of $<4$ joints beyond >six months. Extended oligoarthritis exhibits implication of $>4$ joints beyond $>$ six months $(5,6)$.

- Rheumatoid Factor (RF) negative polyarthritis is designated as arthritis incriminating $\sim 5$ or $>5$ joints within six months of disease occurrence in association with non reactive immunoglobulin M Rheumatoid Factor (IgM RF).Small and large joints are involved in a symmetrical fashion $(5,6)$. Morning stiffness, fatigue and low grade fever accompany joint involvement $(5,6)$. Rheumatoid Factor (RF) positive polyarthritis is exemplified as arthritis implicating $\sim 5$ or $>5$ joints with six months of disease occurrence along with reactive Immunoglobulin M Rheumatoid Factor (IgM RF), as delineated by minimally two evaluations made three months apart. Symmetrical implication of small and large joints is observed along with subcutaneous nodules, fusion of cervical spine, chronic uveitis and destructive hip disease $(5,6)$.

- Systemic arthritis is delineated as arthritis associated with or preceded by fever of around 2 week duration in association with singular or multiple clinical manifestations such as evanescent erythematous rash, generalized lymphadenopathy, hepatomegaly and/or splenomegaly, serositis as described by pericarditis, pleuritis or peritonitis $(5,6)$. Arthralgia is commonly encountered in preliminary systemic Juvenile Idiopathic Arthritis (sJIA). Arthritis may incriminate several joints such as the wrist, knee or ankle. Finger, hip, temporomandibular joint or cervical spine may be occasionally affected, unlike oligoarticular and polyarticular subtypes of juvenile arthritis $(5,6)$. The variant is also designated as systemic- onset juvenile idiopathic arthritis or Still's disease. A variant with severe disease, systemic arthritis occurs in early childhood with equivalent gender distribution. Several extra-articular clinical manifestations such as spiking pyrexia, cutaneous rash upon thoracic cavity or thighs, lymphadenopathy, hepatosplenomegaly, polyarthritis, pericarditis, pleuritic infection, peptic ulcer, hepatitis, anaemia, anorexia, weight loss, myalgia, myositis, tenosynovitis and skeletal 
growth disorders ensue. Disease alleviation is observed in an estimated $50 \%$ children $(5,6)$. Musculoskeletal system depicts symptoms such as joint pain and soft tissue pain arising within the paediatric population, possibly traumatic in nature $(5,6)$.

- Psoriatic arthritis is designated as chronic arthritis associated with psoriasis. Psoriatic variant characteristically depicts arthritis along with psoriasis and minimally two clinical manifestations comprised of inflammation and swelling of an entire finger or toe (dactylitis), pitted nails, onycholysis and family history of psoriasis occurring in a first-degree relative. Uveitis may also be discerned. Aggressive therapy with immunosuppressive agents is recommended $(5,6)$.

- Enthesitis related juvenile idiopathic arthritis typically displays inflammation of enthesis or attachments of tendon, ligament or joint capsule to the bone. Enthesitis commonly occurs within the Achilles' tendon, vertebral column or knee. Incriminated children depict arthritis and enthesitis or may singularly display arthritis or enthesitis along with minimally two clinical manifestations enunciated as inflammation or pain of lumbosacral vertebral column, reactive HLA-B27, discernible family history of ankylosing spondylitis, Enthesitis Related Arthritis (ERA), inflammation or pain within sacro-iliac joint, inflammatory bowel disease, Reiter's syndrome, reactive arthritis or acute anterior uveitis in a first-degree relative and polyarticular or pauciarticular arthritis occurring in male subjects beyond $>6$ years $(5,6)$.

- Undifferentiated arthritis is denominated as chronic arthritis which lacks aforementioned diagnostic criterion in a singular subtype or demonstrates diagnostic criterion associated with $\sim 2$ or $>2$ subtypes $(5,6)$. Juvenile idiopathic arthritis may engender leg length discrepancy and joint contractures. Macrophage activation syndrome may arise in an estimated $10 \%$ subjects due to unchecked activation and proliferation of $\mathrm{T}$ lymphocytes and macrophages. Additionally, growth retardation, amyloidosis, reduced bone mineral density pertinent to chronological age and significant hip involvement which necessitates hip prosthesis may ensue $(5,6)$.

\section{RHEUMATOID ARTHRITIS}
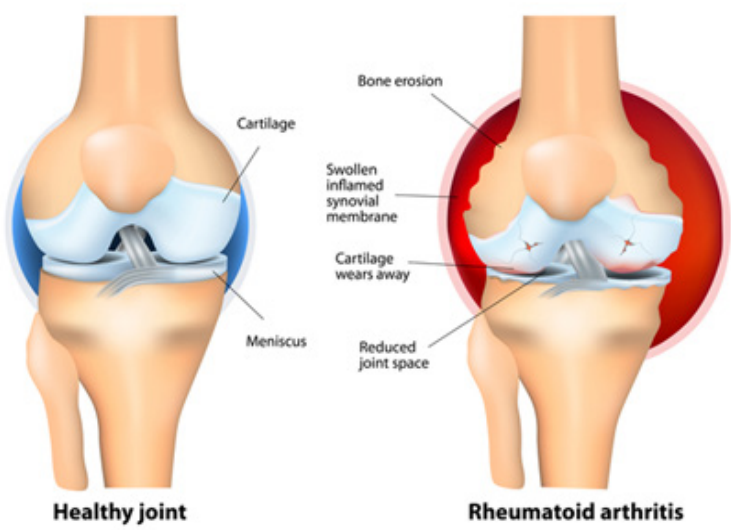

Figure 1: Juvenile rheumatoid arthritis with synovial thickening and inflammation, cartilage and bone erosion and decimated joint space.

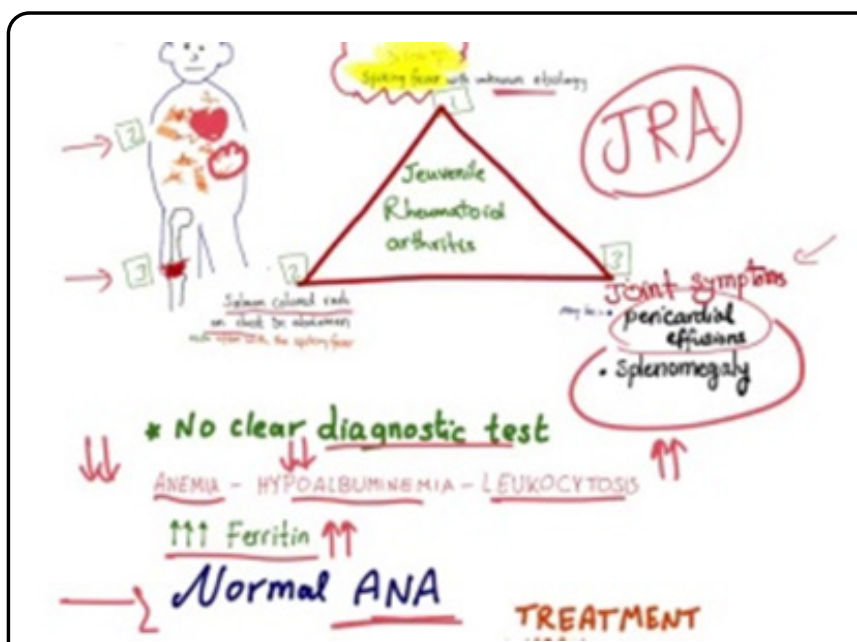

Figure 2: Juvenile rheumatoid arthritis demonstrating diverse clinical symptoms and non specific biochemical and haematological investigations.

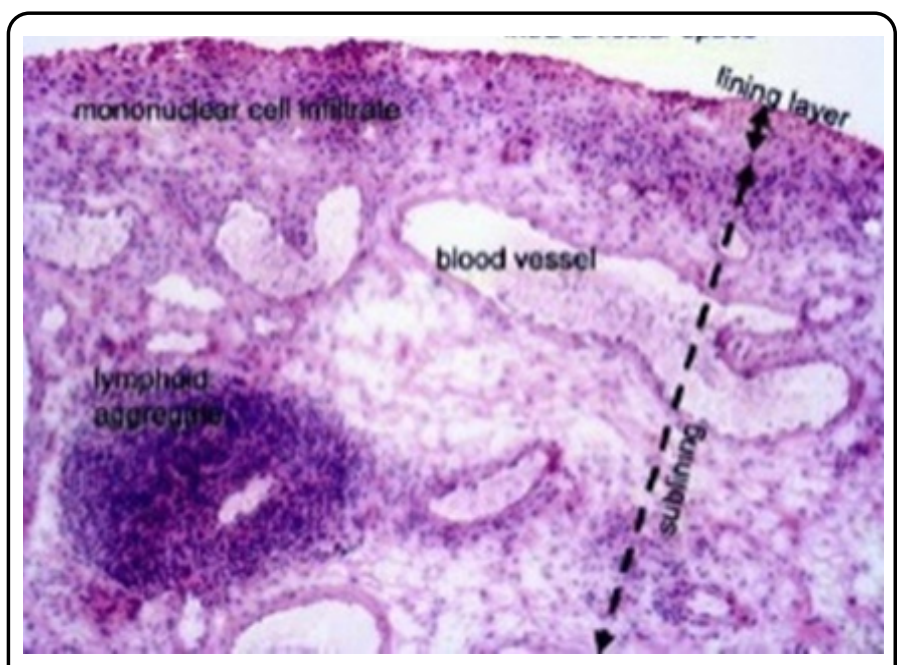

Figure 3: Juvenile rheumatoid arthritis exhibiting hyperplastic synovial epithelium commingled with chronic inflammatory infiltrate of lymphocytes and histiocytes and patent vascular articulations.

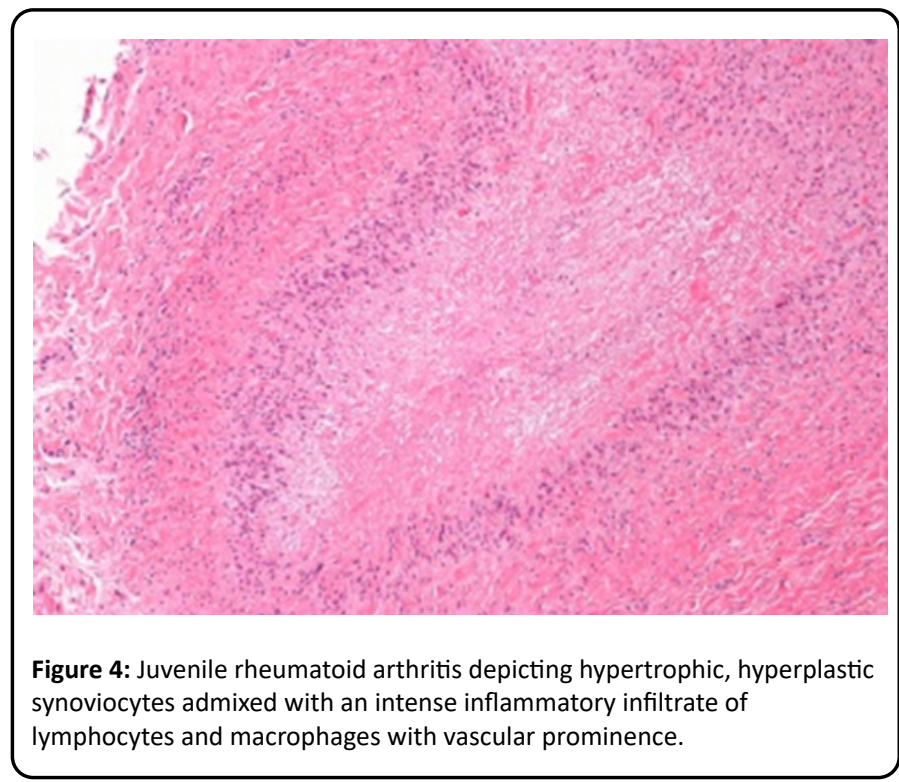




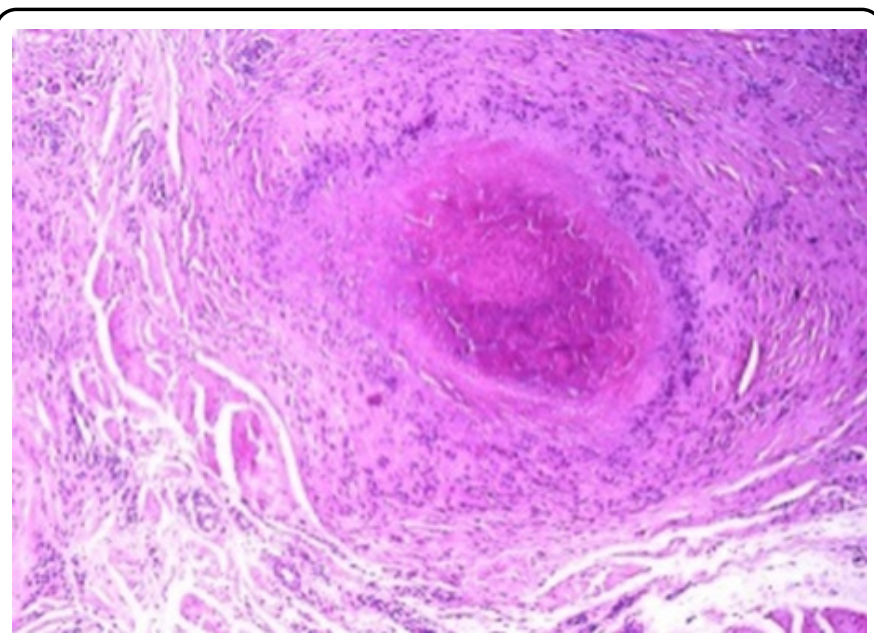

Figure 5: Juvenile rheumatoid arthritis exemplifying a rheumatoid nodule with intense infiltration of chronic inflammatory cells along with synovial hyperplasia, hypertrophy and vascular articulations.

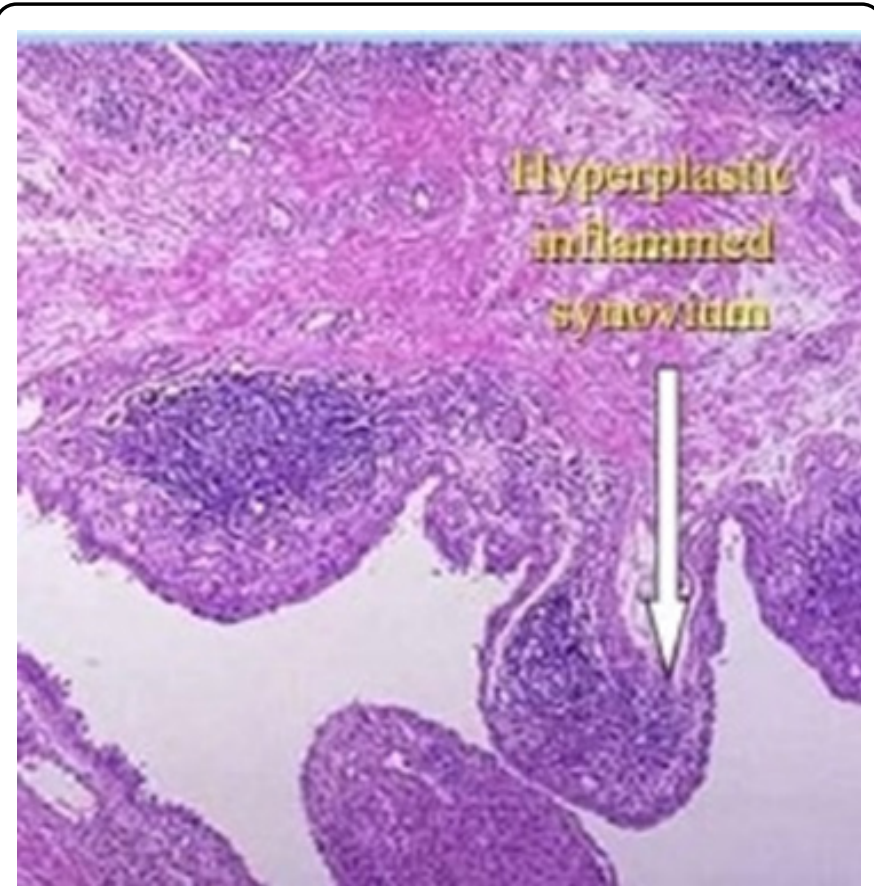

Figure 6: Juvenile rheumatoid arthritis exhibiting synovial hyperplasia, proliferation and infiltration of chronic lymphocytic infiltrate with configuration of pannus.

\section{Differential Diagnosis}

As juvenile idiopathic arthritis is a diagnosis of exclusion, the condition necessitates a segregation from

- Oligoarthritis requires a demarcation from poststreptococcal reactive arthritis, Lyme arthritis, acute rheumatic fever, non specific reactive arthritis, toxic synovitis, septic arthritis, pyomyositis, steroid-induced osteonecrosis, sickle cell disease, haemophilia, scurvy, osteomyelitis, Chronic Nonbacterial Osteomyelitis (CNO), sports induced injury, non-accidental joint injury, Pigmented Villonodular Synovitis (PVNS), osteoid osteoma, bone tumours, neuroblastoma, leukaemia and lymphoma $(7,8)$.

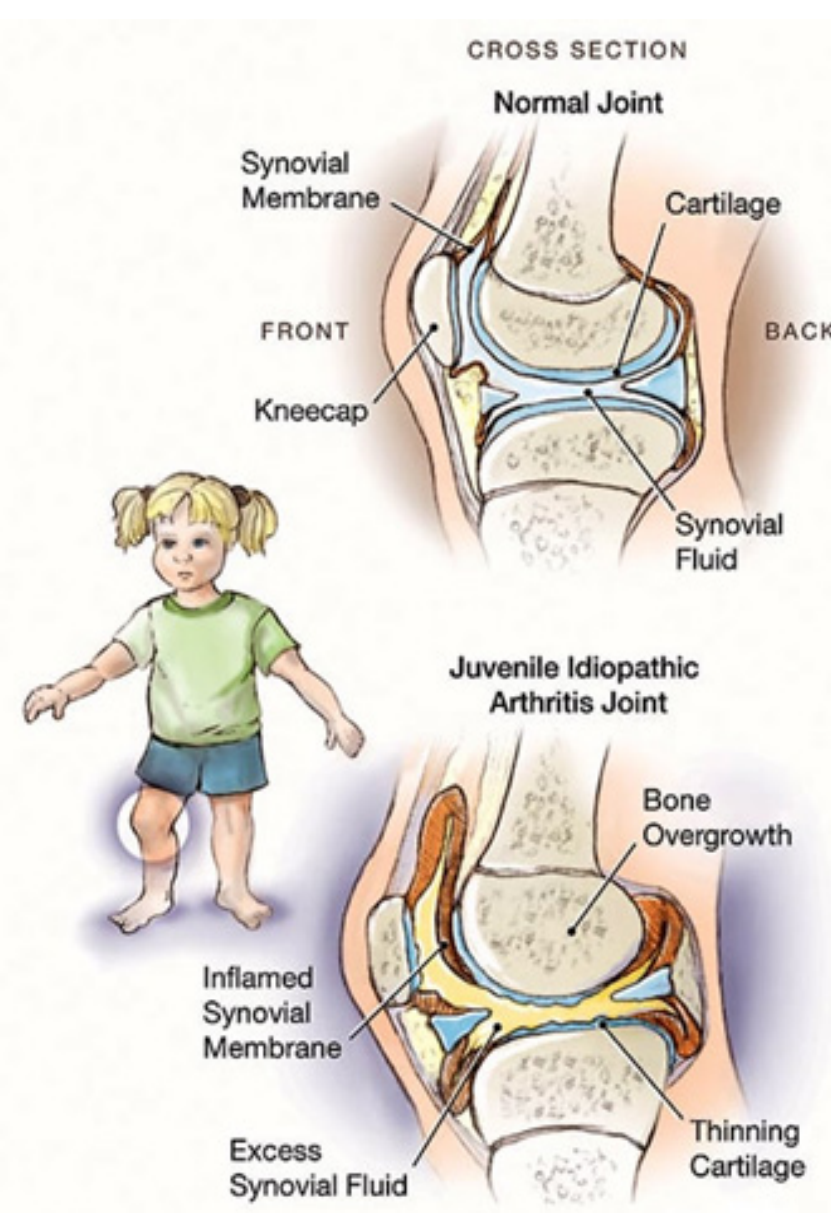

Figure 7: Juvenile idiopathic arthritis depicting synovial inflammation and proliferation, thinned out cartilage and bony overgrowth.

- Polyarthritis requires a distinction from post-streptococcal reactive arthritis, Lyme arthritis, acute rheumatic fever, non specific reactive arthritis, scurvy, Chronic Nonbacterial Osteomyelitis (CNO) or chronic recurrent multifocal osteomyelitis, non-accidental joint injury, Systemic Lupus Erythematosus (SLE), Mixed Connective Tissue Disorder (MCTD), Sjögren's syndrome, scleroderma, sarcoidosis, Blau syndrome, arthritis associated with inflammatory bowel disease, Farber's disease, benign hypermobility joint syndrome and amplified musculoskeletal pain syndrome $(7,8)$.

- $\quad$ Systemic arthritis necessitates a segregation from infections such as mycoplasma, cat scratch disease, bacterial endocarditis, Lyme disease, acute rheumatic fever, periodic fever syndrome, aphthous stomatitis, pharyngitis and cervical adenitis (PFAPA syndrome), auto-inflammatory conditions, systemic vasculitis as encountered in polyarteritis nodosa or Kawasaki's disease, inflammatory bowel disease, malignant disorders such as leukaemia, lymphoma, neuroblastoma and Castleman's disease $(7,8)$.

- Enthesitis-related arthritis mandates a segregation from apophysitis as encountered in Osgood-Schlatter disease or Sever's disease, Inflammatory Bowel Disease (IBD), Chronic Recurrent Multifocal Osteomyelitis (CRMO) or amplified musculoskeletal pain syndrome. Additionally, 
differentiation is required from osteomyelitis, psoriatic arthritis, leukaemia, Caffey disease, chronic eosinophilic granuloma, osteoporosis and involutional osteoarthritis $(7,8)$.

\section{Investigative Assay}

Although diverse imaging modalities are unsatisfactory for investigating juvenile idiopathic arthritis, manifestations such as joint deterioration, fracture, neoplasms, infection or congenital skeletal anomalies may be adequately assessed. Generally, plain radiographs, bone scan, Computerized Tomography (CT), magnetic resonance imaging (MRI) and Duel-Energy X-Ray Absorptiometry (DEXA) scan is recommended $(8,9)$. Additionally, investigations such as arthrocentesis and synovial tissue sampling can be adopted $(8,9)$.

Juvenile idiopathic arthritis is appropriately discerned contingent to pertinent clinical and family history, symptoms, number of incriminated joints, imaging features and haematological, biochemical or serological parameters $(8,9)$.

Comprehensive clinical history, age of disease onset, implicated joints, duration of arthritis, associated clinical symptoms, accompanying disorders, general physical and pertinent musculoskeletal examination is essential for appropriate discernment and classification of juvenile idiopathic arthritis $(9,10)$. Haematological, biochemical and serological parameters such as Complete Blood Count (CBC), Erythrocyte Sedimentation Rate (ESR), C- Reactive Protein (CRP), Antinuclear Antibodies (ANA), Rheumatoid Factor (RF), Anti-Cyclic Citrullinated Peptide (anti-CCP) antibodies and human leukocyte antigen B-27(HLA-B27) require evaluation $(9,10)$. Typical inflammatory markers are reactive in oligoarthritis.

Reactive Rheumatoid Factor (RF) or Anti-Cyclic Citrullinated Peptide (anti-CCP) antibodies are usually not beneficial although indicate an inferior biological course and disease outcome $(9,10)$. Serum ferritin, fibrinogen, Aspartate Aminotransferase (AST) and triglyceride is recommended for assessment of Macrophage Activation Syndrome (MAS). Exclusion of pertinent, identical diseases may be mandated for cogent evaluation $(9,10)$.

Preliminary stage of juvenile idiopathic arthritis may be undiscernible with plain radiography. Plain radiography is the initial imaging modality employed for assessing joints with distinctive clinical symptoms. Plain radiography ameliorates cogent disease discernment, assesses joint injury in juvenile idiopathic arthritis and provides a precise differential diagnosis. Heterogeneous clinical manifestations necessitate multimodal imaging $(9,10)$.

Soft tissue manifestations of arthritis upon radiography appear as soft tissue swelling, soft tissue density and dislocation of adipose tissue. Additionally, periarticular osteoporosis, narrowing of joint space, erosion and deformity of bone and joint subluxation or ankyloses of incriminated joint may be delineated in advanced disease $(9,10)$.

Ultrasonography (US) is an accessible, non-irradiative investigative modality employed to assess juvenile idiopathic arthritis $(9,10)$.

Ultrasonography permits analogy with contralateral joint, dynamic evaluation of joints and guides intra-articular corticosteroid injections. Ultrasonography can appropriately assess synovial thickening, joint effusion, tenosynovitis, enthesitis and bone erosion. Evaluation of synovial thickening and synovitis is especially significant for cogent disease discernment. Aforesaid features manifest as anomalous hypoechoic tissue associated with joint lines or circumscribing tendons. Magnetic Resonance Imaging (MRI) is the optimal modality for evaluating synovitis accompanying juvenile idiopathic arthritis. Bones and soft tissue of inflamed joints can be examined within comprehensive planes and with superior contrast resolution. MRI can accurately discern bone marrow oedema and bone erosion $(10,11)$.

\section{Therapeutic Options}

Objectives of medical management are pain alleviation, joint preservation with conserved joint mobility and function, achieving normal skeletal growth, decimation of associated systemic symptoms and joint deterioration along with circumventing progression of inflammation and disease $(10,11)$. Clinical symptoms may be ameliorated by application of hot or cold packs, paraffin wax packs, warm baths, gentle range of motion (ROM), enhanced physical activity or exercises such as swimming, maintenance of healthy weight and muscle relaxation $(10,11)$. Pertinent physical therapies affect muscular tone, ensure muscle strengthening, range of motion (ROM), muscular stretching, joint protection, pain reduction and unsupervised exercises. Employment of splints or orthotics may be beneficial in maintaining normal bone and joints and circumvent deformities during skeletal growth $(10,11)$. Juvenile idiopathic arthritis is optimally managed with anti-inflammatory and immunomodulatory drugs. Pertinent pharmacological agents employed are contingent to disease subtype, disease severity, specific joint deterioration, associated diseases and individual acceptance of particular therapy $(10,11)$.

Aggressive medication is recommended for treating juvenile idiopathic arthritis such as Disease-Modifying AntiRheumatic Drugs (DMARDs) as methotrexate in addition to sulfasalazine, immuno-suppressants, Tumour Necrosis Factor (TNF) inhibitors, Non Steroidal Anti-Inflammatory agents (NSAIDs), corticosteroids or analgesics. Nonsteroidal AntiInflammatory Drugs (NSAIDs) are predominantly employed for treating preliminary symptoms emerging in diverse subtypes. Additionally, contemporary, aggressive molecules such as methotrexate and biologics as the anti cytokine agents may be utilized. Novel therapeutic agents can prohibit biological mechanisms inducing persistent inflammation of selective joints $(10,11)$. Physical therapy, nutritional and psychosocial assistance along with surgical intervention may be adopted in pertinent, severe instances. Preliminary, precise disease discernment and therapy is mandated in order to circumvent permanent joint deterioration and conserve joint function, enhance percentage of disease remission and ameliorate extended disease prognosis $(10,11)$.

\section{Conclusion}

Juvenile idiopathic arthritis requires a segregation from post-streptococcal reactive arthritis, Lyme arthritis, acute rheumatic fever, non specific reactive arthritis, toxic synovitis, septic arthritis, pyomyositis, steroid-induced osteonecrosis, sickle cell disease, haemophilia, scurvy, Chronic Nonbacterial 
- jteomyelitis (CNO), sports induced injury, non-accidental joint injury, Pigmented Villonodular Synovitis (PVNS), osteoid osteoma, bone tumours, neuroblastoma, leukaemia, lymphoma, Systemic Lupus Erythematosus (SLE), Mixed Connective Tissue Disorder (MCTD), Sjögren's syndrome, scleroderma, sarcoidosis, Blau syndrome, arthritis associated with Inflammatory Bowel Disease (IBD), Farber's disease, benign hypermobility joint syndrome, amplified musculoskeletal pain syndrome, mycoplasma, cat scratch disease, bacterial endocarditis, periodic fever syndrome, aphthous stomatitis, Pharyngitis And Cervical Adenitis (PFAPA syndrome), auto-inflammatory conditions, systemic vasculitis as encountered in polyarteritis nodosa or Kawasaki's disease, Castleman's disease, apophysitis as encountered in Osgood-Schlatter disease or Sever's disease, Chronic Recurrent Multifocal Osteomyelitis (CRMO), psoriatic arthritis, Caffey disease, chronic eosinophilic granuloma, osteoporosis and involutional osteoarthritis. Magnetic resonance imaging (MRI) is the optimal modality for evaluating synovitis accompanying juvenile idiopathic arthritis. Juvenile idiopathic arthritis can be treated with Disease-Modifying AntiRheumatic Drugs (DMARDs) as methotrexate, sulfasalazine, immuno-suppressants, Tumour Necrosis Factor (TNF) inhibitors, Non Steroidal Anti-Inflammatory Agents (NSAIDs), corticosteroids or analgesics.

\section{References}

1. Thatayatikom A, Modica R, De Leucio.A (2021) Juvenile Idiopathic Arthritis. Stat Pearls International,Treasure Island, Florida.

2. Martini A, Ravelli A, Avcin T, et al. (2019) Toward New Classification Criteria for Juvenile Idiopathic Arthritis: First Steps, Paediatric Rheumatology International Trials Organization
International Consensus. J Rheumatol 46(2): pp. 190-197.

3. Horton DB, Shenoi S (2019) Review of environmental factors and juvenile idiopathic arthritis" Open Access Rheumatol 11(11): pp. 253-267.

4. Rigante D, Bosco A, Esposito S (2015) The Etiology of Juvenile Idiopathic Arthritis. Clin Rev Allergy Immunol 49(2): pp. 253261.

5. Giancane G, Alongi A, Ravelli A (2017) Update on the pathogenesis and treatment of juvenile idiopathic arthritis. Curr Opin Rheumatol 29(5): pp. 523-529.

6. Cobb JE, Hinks A, Thomson W (2014) The genetics of juvenile idiopathic arthritis: current understanding and future prospects. Rheumatology 53(4): pp. 592-599.

7. Cimaz R (2016) Systemic-onset juvenile idiopathic arthritis" Autoimmun Rev 15(9): pp. 931-934.

8. Brown RA, Henderlight M, Do T, et al. (2018) Neutrophils From Children With Systemic Juvenile Idiopathic Arthritis Exhibit Persistent Proinflammatory Activation Despite Long-Standing Clinically Inactive Disease. Front Immunol 9(3): pp. 2995.

9. Maggi L, Mazzoni A, Cimaz R, et al. (2019) Th17 and Th1 Lymphocytes in Oligoarticular Juvenile Idiopathic Arthritis" Front Immunol 10(3): pp. 450.

10. Malattia C, Rinaldi M, Martini A, et al. (2018) The role of imaging in juvenile idiopathic arthritis. Expert Rev Clin Immunol 14(8): pp. 681-694.

11. Barut K, Adrovic A, Sahin S, et al. (2019) Prognosis, complications and treatment response in systemic juvenile idiopathic arthritis patients: A single-center experience" Int J Rheum Dis 22(9): pp. 1661-1669. 